\title{
SCHASKOS: A SKOS-Based Tool for Supporting the Decision Making Process in a Supply Chain
}

\author{
Daniel Vázquez-Ramírez, Cristian Aaron Rodríguez-Enríquez, \\ Giner Alor-Hermández, Cuauhtémoc Sanchez-Ramírez, \\ María Antonieta Abud-Figueroa, Luis Ángel Reyes-Hernández \\ Instituto Tecnológico de Orizaba, \\ División de estudios de posgrado e investigación, Veracruz, \\ Mexico \\ danielvazquezrmz@outlook.com, \{crodriguezen, 1.a.reyes.h\}@gmail.com, \\ \{galor, csanchez, mabud\}@itorizaba.edu.mx
}

\begin{abstract}
Semantic Web technologies provide innovative means for organizing, describing, and managing digital resources in a wide range of formats. From this perspective, Linked Data has recently gained some attention in the supply chain management field as an effective way to provide and share data across all the value chain. Semantic Web technologies can support the seamless integration of data, on-the-fly composition and interoperation of Web services, as well as more intuitive search engines. This search engines allows the supply chain data exploitation in order to get valuable knowledge. However, a successful implementation and use of Semantic Web technologies requires both information professionals and system developers. In this paper, we propose a Web-based tool called SCHASKOS that covers these needs by working with the W3C's Simple Knowledge Organization Systems (SKOS) by using Linked Data. The SCHASKOS tool is a Linked Data-based tool for non-experience information professionals that allows the SKOS and Linked Data adoption for supply chain knowledge management in organizations. Additionally, a case study of how the SCHASKOS tool works and their benefits are presented.
\end{abstract}

Keywords: Linked data, semantic web, SKOS, supply chain.

\section{Introduction}

The knowledge management in organizations has gained momentum during the last decade as many academic; executives and practitioners have recognized that applying knowledge will become the key competitive factor and the most valuable and strategic resource in their companies. Knowledge is the result of a process where an individual makes contact with data found in various kinds of "data sources" such as reports, letters, bills, memos, e-mails, notes and software to mention but a few [1]. Then this individual adds value to these "data sources" to convert data into information, and finally the information translates into knowledge when it is used for improving a process. In other context, supply chain management is an important area of research. Under the context of coordinating the supply chain, several Knowledge Management approaches have 
been developed to extent supply chains, rather than single companies, are now seen as interoperable units of a value chain. The present and the future of the Knowledge Management in organizations is the exploitation of computational systems that are able to store, process and transmit knowledge from one unit to another in the value chain, in order to daily facilitate operations within an organization, and especially timely detect problems, and possible solutions. From this perspective, SKOS is a family of formal languages designed to represent thesauri, taxonomies, subject-heading systems, or any other type of structures of controlled vocabulary for the Semantic Web. These formal languages allow improving the Knowledge Management in a powerful semantic way. Taking into consideration that knowledge is an effective way to improve processes in organizations; in this paper we explore the use of SKOS for knowledge management of a supply chain.

This paper is organized as follows: Section 2 introduces a literature-review of tools for SKOS visualization, SKOS vocabularies transformations, and other related works with knowledge management in supply chains. The SCHASKOS architecture is presented in Section 3. In Section 4, a case study is presented. Finally, conclusions and future work are presented in Section 5.

\section{State of the Art}

Nowadays, they are different knowledge domains has been used SKOS as a mechanism for knowledge management in several research fields such as geography [2-3] and medical sciences [5], to mention but a few. However, in supply chain management field, the use of SKOS for knowledge management has not been reported before. In this section, we describe several related works to SKOS, these works were selected according to the following classifications: 1) visualization tools and 2) vocabularies transformations. Also, a review of related works with the knowledge management in supply chain is presented.

There are several SKOS visualization tools reported in the literature, for instance GeoNetwork [2] and ThManager [3], these tools have been used in the geography domain. In the GeoNetwork and ThManager research works, a multi-platform desktop and open source application were presented. GeoNetwork allows importing a thesauri in SKOS-based format and locate geospatial data through an interactive map. GeoNetwork support ISO standards for thesaurus creation such as: ISO 19115, ISO 19119 and ISO 19110 by using the ISO 19139, FGDC and Dublin Core.

In [4], ThManager was used to transform the Thesaurus for French Local Archives to SKOS-based format. Moreover, with ThManager an application for using the Thesaurus for French Local Archives was developed.

In [5] the authors presented a Web-based tool for the medical domain that allows the graphical visualization of the concepts contained in a SKOS vocabulary, and the different relationships among labels of each concept.

Vocbench [6] is a Web-based tool that supports an aquatic science vocabulary and a fishing thesaurus. Vocbench used the SPARQL query language and it was used to manage the AGROVOC thesauri. 
SISSVoc [7] is a Linked Data API that allows the access to published vocabularies, which use the SKOS-based format. SISSVoc is used in a large number of projects, which mainly belongs to environmental sciences.

SKOS Play [8] is an open source web-based tool that allows the visualization of thesaurus, taxonomies, and controlled vocabularies expressed in SKOS. Another interesting tool for vocabulary management is Pool Party, a commercial Web-based tool that allows publishing thesaurus as Linked Open Data. The latest Pool Party version allows performing a vocabulary quality report through "The PoolParty SKOS Quality Checker". The quality checker is based on qSKOS (quality simple Knowledge Organization System).

TopBraid Enterprise Vocabulary Net [9] is a commercial Web-based tool that allows managing business vocabularies, which is mainly developed to business domain. In addition, TopBraid Enterprise Vocabulary Net allows to organizations the possibility of creating and editing ontologies or SKOS-based business vocabularies.

With regard to the knowledge management in supply chains, a literature review to supply chain is presented in [10], the objective of this review was to provide a taxonomy developed about supply chain management field used to assist in the search ranking in the supply chain field in order to provide a framework for categorizing related literature in supply chain management according to two criteria: content-oriented (level of analysis and element of the exchange within the studied network) and methodologyoriented (theoretical, empirical, prescriptive and descriptive).

In [11] the authors conducted a study of knowledge management in supply chain networks. Additionally, a knowledge management in supply chain conceptual model was proposed as an extension of Nonaka and Takeuchi model.

In [12] a literature review of supply chain was presented to assess the relationship between knowledge management and supply chain management. The literature review showed that the knowledge management is considered a tool for the supply chain integration. Additionally, the lack of ITs (Information Technologies) integration to improve knowledge sharing among supply chain was discussed.

In [13] a scientific framework to solutions adoption of knowledge management in a supply chain was presented. This framework used a hybrid multi criteria technique, which combined fuzzy Analytical Hierarchy Process (AHP) and fuzzy Technique for Order Performance by Similarity to Ideal Solution (TOPSIS).

In [14] a platform for knowledge sharing in a supply chain by using semantic Web technologies was proposed. The platform involves a semi-structured model to represent knowledge, an agent-based annotation process to resolve issues associated with the heterogeneity of knowledge documents. Additionally, an articulation mechanism to improve the effectiveness of interoperability by using two ontologies was presented.

In [15] a classification of knowledge in a supply chain was presented, identifying various kinds of knowledge and their definition. The classification was developed in five categories depending on the stages in a supply chain: (1) planning, (2) production, (3) storage, (4) delivery, and (5) transportation. As part of the research a case study of a food supply chain was defined. In Table 1, a summary about the state of art is presented. We have considered if the work is a SKOS-based tool (SKOS), Linked Data (LD) is used, and finally, if the research work was developed for Supply Chain Management (SCM). 
Table 1. State of the art highlights.

\begin{tabular}{lccc}
\hline \multirow{2}{*}{\multicolumn{1}{c}{ Research Work }} & \multicolumn{3}{c}{ Feature } \\
\cline { 2 - 4 } & SKOS & $\begin{array}{c}\text { Linked } \\
\text { Data }\end{array}$ & $\begin{array}{c}\text { Supply Chain } \\
\text { Management }\end{array}$ \\
\hline Open Source Geospatial Foundation [2] & Y & N & N \\
Lacasta Javier, et al [3] & Y & N & N \\
de Grimoüard-Sibille [4] & Y & N & N \\
Conway Mike, et al [5] & Y & N & N \\
Agricultural Information & Y & Y & N \\
Management Standards [6] & & & N \\
JD Cox Simon [7] & Y & Y & N \\
Francart Thomas [8] & Y & N & N \\
Schandl Thomas, et al & Y & N & N \\
Morshed Ahsan, et al [9] & Y & N & Y \\
Croom Simon, et al [10] & N & N/A & Y \\
Samuel Karine Evrard, et al [11] & N & N & Y \\
Marra Marianna, et al [12] & N & N & Y \\
Patil Sachin K, et al [13] & N & N & Y \\
Huang Chun-Che, et al [14] & N & N & Y \\
Almuiet Mohammad Zayed, et al [15] & N & N & \\
\hline
\end{tabular}

\section{SCHASKOS Architecture}

Our Knowledge Organization System called SCHASKOS has a layered design in order to organize its components. This layered design allows scalability, easy maintenance and multi-device presentation capabilities, because its task and responsibilities are distributed.

\subsection{Architecture Description}

The general architecture is shown in Figure 1. Each component of SCHASKOS has a function explained as follows:

Data layer: This layer stores concepts (SKOS), social network data; data source URIs for Linked Data and non-structured data (files and raw data) for knowledge management. This layer additionally contains all the configuration tables allowing the operation of the modules and services offered by SCHASKOS.

Data Access layer: This layer communicates with the Data Access layer and it contains the representation on flat objects of each relationship of the database management system (for relational data), a XML-based transformation schema for both nonstructured data and social network data; and finally a SKOS data manager. 
Core layer: This layer is responsible for interpreting data from the Data Access layer through the Linked Data engine and SKOS engine, and it builds a XML-based representation. This layer additionally allows the data knowledge management and its representation.

Service layer: This layer provides a set of services (modules) offered by SCHASKOS. These services represent different functions such as visualization, analysis, search, storage and data editing. This layer communicates with the data layer to obtain the required content at any time. In this layer, a module is located responsible for the representation of diagrams with different concepts in SKOS-based format obtained.

Integration layer: This layer allows the creation of informative diagrams, request management, and it determines the way to get the information and build the diagram requested by the user in the presentation layer.

Presentation layer: In this layer, SCHASKOS determines the diagram to be presented to the final user. This layer represents the view that the user has of the system by using HTML5 and CSS3. The presentation layer unknown events taken by SCHASKOS, it only gets the information and required diagrams to display to the end user in a graphical user interface.

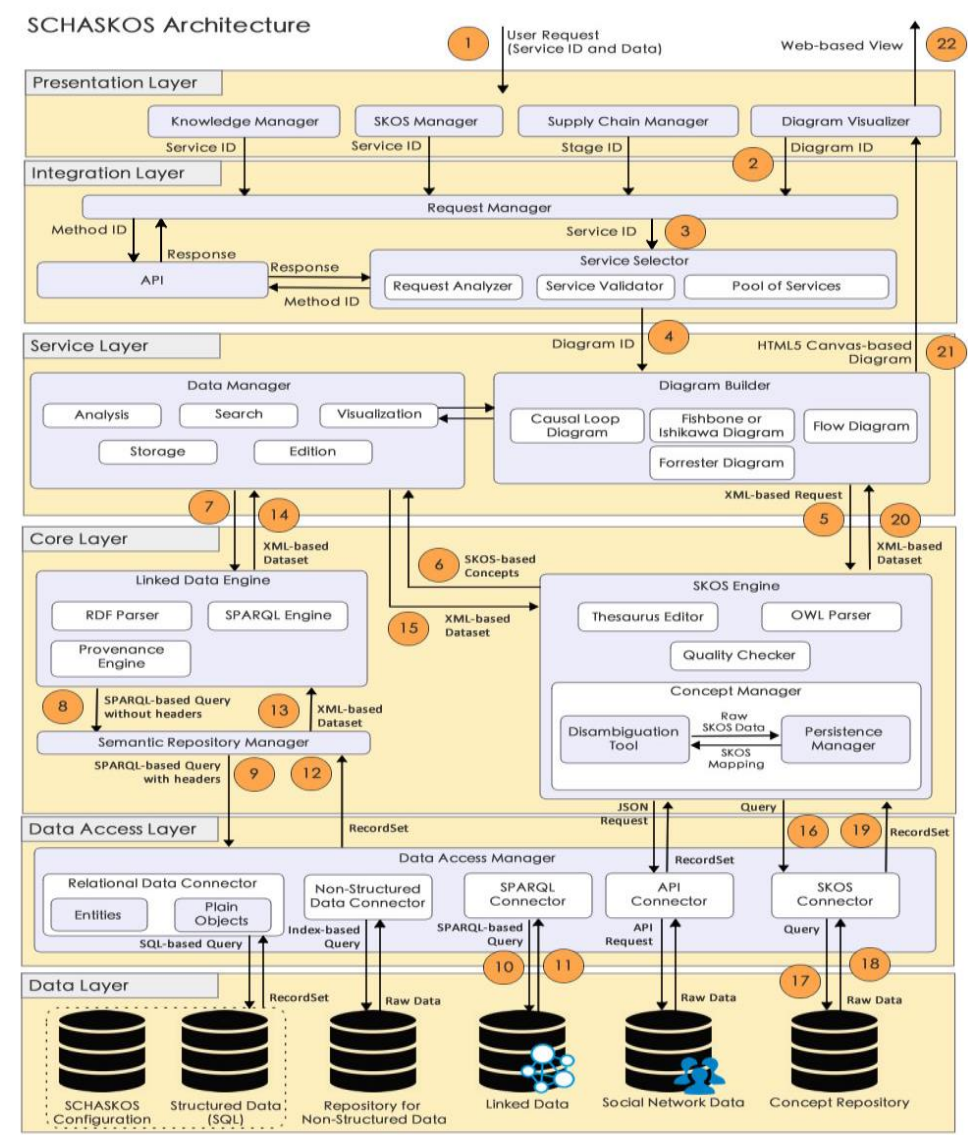

Fig. 1. SCHASKOS architecture. 


\subsection{Component Description}

In SCHASKOS architecture, each component has a specific function that is explained in Table 2.

Table 2. Component Description.

\begin{tabular}{|c|c|}
\hline Component & Functionality \\
\hline Linked Data & $\begin{array}{l}\text { This component represents the Linked Data cloud, which contains } \\
\text { information available on various domain fields. }\end{array}$ \\
\hline $\begin{array}{l}\text { Concept } \\
\text { Repository }\end{array}$ & $\begin{array}{l}\text { This component is an extensive vocabulary related to the supply chain } \\
\text { management. }\end{array}$ \\
\hline $\begin{array}{l}\text { SPARQL } \\
\text { Connector }\end{array}$ & $\begin{array}{l}\text { This component allows performing SPARQL-based queries over the } \\
\text { Linked Data cloud by using a SPARQL endpoint. }\end{array}$ \\
\hline SKOS Connector & $\begin{array}{l}\text { The SKOS connector allows the access to the concept repository, } \\
\text { which is requested by the SKOS Engine module for related concepts. }\end{array}$ \\
\hline $\begin{array}{l}\text { Linked Data } \\
\text { Engine }\end{array}$ & $\begin{array}{l}\text { This module contains a component to analyze RDF data because SKOS } \\
\text { is RDF-based. Additionally, a SPARQL Engine is used to analyze the } \\
\text { data provenance. }\end{array}$ \\
\hline SKOS Engine & $\begin{array}{l}\text { This component provides four sub-components, these components } \\
\text { includes: 1) The concept manager module, 2) The disambiguation tool } \\
\text { that identifies the most appropriate meaning among the concepts, 3) } \\
\text { The persistence manager that is responsible of storing the knowledge in } \\
\text { SKOS-based format according the information obtained in a XML- } \\
\text { based file, and 4) The SKOS Mapping provides tags used to indicate } \\
\text { the relation degree among the builder concepts and previously stored } \\
\text { concepts by the persistence manager. }\end{array}$ \\
\hline Data Manager & $\begin{array}{l}\text { This module contains different operations that the system executes with } \\
\text { the information provided by the user, such as edit, search, analyze, } \\
\text { store and display. }\end{array}$ \\
\hline Diagram Builder & $\begin{array}{l}\text { This module allows building diagrams to analyze related information in } \\
\text { a supply chain, in order to find problems and the expert takes a } \\
\text { decision. SCHASKOS allows presenting the following diagrams to the } \\
\text { final user: 1) Causal Loop Diagram, shows the structure and } \\
\text { relationships between different variables in a system. This kind of } \\
\text { diagram allows knowing the reasons why a variable can affect another, } \\
\text { 2) Fishbone or Ishikawa Diagram, proposed by dr. Kaorou Ishikawa, } \\
\text { allows graphically showing the causes of a problem, allowing a } \\
\text { thorough analysis, and 3) Forrester Diagram, created by Jay Forrester, } \\
\text { is used to observe the evolution of the variables through various } \\
\text { equations that allows validate the model obtained in the causal loop } \\
\text { diagram. }\end{array}$ \\
\hline Request Manager & This module manages the requests made by the final user. \\
\hline Request Analyzer & This component identifies the entire user's request. \\
\hline Service Validator & $\begin{array}{l}\text { This component, validate that the request service is valid and available } \\
\text { for the user. }\end{array}$ \\
\hline Pool of services & This component includes all services of the SCHASKOS tool. \\
\hline
\end{tabular}


SCHASKOS: A SKOS-Based Tool for Supporting the Decision Making Process in a Supply Chain

\begin{tabular}{ll}
\hline \multicolumn{1}{c}{ Component } & \multicolumn{1}{c}{ Functionality } \\
\hline API & $\begin{array}{l}\text { This component allows the access to the methods available in } \\
\text { SCHASKOS through a public interface. }\end{array}$ \\
$\begin{array}{l}\text { Diagram } \\
\text { visualizer }\end{array}$ & This module allows the user to view the built diagram by SCHASKOS. \\
\hline
\end{tabular}

\subsection{Workflow Description}

The workflow describes the functionality of SCHASKOS's architecture. The workflow is explained as follows:

(1) the user makes a request to SCHASKOS using a Web browser by indicating a URL,

(2) the Presentation Layer identifies the user's request and redirects the HHTP-based request to the Integration Layer where the HTTP-based request is analyzed by the Request Manager Component to determine what type of service is being requested,

(3) the Integration Layer uses the Service Selector Component to determine whether the HTTP request is valid. If a request for a valid service has been requested, the Service Selector Component is prepared to invoke the appropriate service from the Service Layer,

(4) if the service is valid and available, a query is carried out to recover the service identifier in the Pool of Services Component provided by SCHASKOS. In the event that the service is not available or is invalid, SCHASKOS returns an error message with the failure reasons and a list of available services,

(5) if the requested service is the generation of a Diagram, the request is redirected to the Diagram Builder Component in the Service Layer where it performs an XML-based request to the SKOS Engine Component through the Core Layer,

(6) the SKOS Engine transform the XML-based request in SKOS format, and redirects the request to the Data Manager Component in the Service layer in order to store the data,

(7) the concepts are directed to Linked Data Engine component in the Core Layer where its origin is identified by using the Engine Provenance Component,

(8) the Core Layer uses the SPARQL Engine component available in the Linked Data Engine module to build and run a query without using headers to find information in the semantic repository, without indicating a source of information,

(9) the semantic Repository Manager module builds a SPARQL-based query by using headers, it is sent to the Data Access Manager through the Data Access Layer,

(10) the Data Access Layer uses the SPARQL Connector component to execute the SPARQL-based query built on the Core Layer on the Linked Data Cloud to locate information from a particular source, (10) if the query returns values, they are sent back to the SPARQL Connector component available from the Data Access Layer,

(11) the Data Access Layer stores the results in a Record Set to be sent to the Semantic Repository Manager component,

(12) the Semantic Repository Manager transforms the result stored in the Recordset to XML-based format to be sent to the Linked Data Engine module,

(13) the Service Layer uses the Data Manager to store the result set in XML-based format sent by the Linked Data Engine, 
(14) the XML-based data set is sent to SKOS Engine module to be transformed to SKOS-based format,

(15) a query is executed to be sent to SKOS connector module available in the Data Access layer for obtained information of the concept repository,

(16) the query is executed on the repository concepts to compare the information obtained with the concepts related to supply chain,

(17) the data obtained are sent to the SKOS Connector module to be stored in a Recordset,

(18) the Recordset with the information obtained is sent to SKOS Engine module to be stored in SKOS-based format,

(19) in order to build the diagram requested by the user, it is necessary to transform the information SKOS to XML-based format, the dataset in XML-based format is sent to the service layer to build the diagram,

(20) the Diagram Builder module is responsible for building the diagram applied to all information obtained using HTML5 Canvas, which is sent to the presentation layer for display to the user. The diagram constructed is displayed to the user using a Web browser, at this point; the user has the opportunity to make decisions.

\section{Case Study: Generating a Ishikawa Diagram to Help the User in the Decision Making Process}

SCHASKOS is a Linked Data-based tool that allows knowledge management in organizations and generating Ishikawa, causal and Forrester diagrams by adopting Linked Data and SKOS to facilitate information management. The main idea of SCHASKOS is to help users better manage their information using the labels provided by SKOS. SCHASKOS transforms this information into a diagram for identifying changes and possible problems occurring within the organization and so, allows the user to make decisions in order to improve the performance of a supply chain. To explain SCHASKOS functionality, the following case study is presented for generating a diagram (Ishikawa or Fishbone, causal and Forrester) in order to help in the decisionmaking process. Let us suppose that an organization dedicated to dairy milk production will keep management for their full supply chain. In this context, there is a constraint: if the administrator of the supply chain is a non-experienced computer user, and therefore does not have the required skills to develop an Ishikawa, causal or Forrester diagram. As a solution to this issue, SCHASKOS provides a set of graphic interfaces that allow any user capture and transform information into a diagram by using SCHASKOS capabilities. To do this, the user must be follow the next steps: (1) the user accesses SCHASKOS via an authentication mechanism by entering a login name and password, (2) once the user has been authenticated, the user selects the option to create a new project and entering a name for the project, (3) The next step is to capture the information of each link in the supply chain, since suppliers have supplied to the organization, changes in raw materials or the method for making products until the reasons for a product return, (4) Once the user has captured all the available information, the next step is to transform it into a diagram to help user in the data analysis The user select the option "Diagram builder" and choose the diagram type, (4) SCHASKOS transform the information obtained in the Linked Data Cloud and the 
concept repository in a diagram. The diagram generated is available in the application interface (this is depicted in Figure 2), and (5) once SCHASKOS has finished the building diagram, the user can view details selecting a cause in the case of Ishikawa diagram. This process is depicted in Figures 2 and 3.

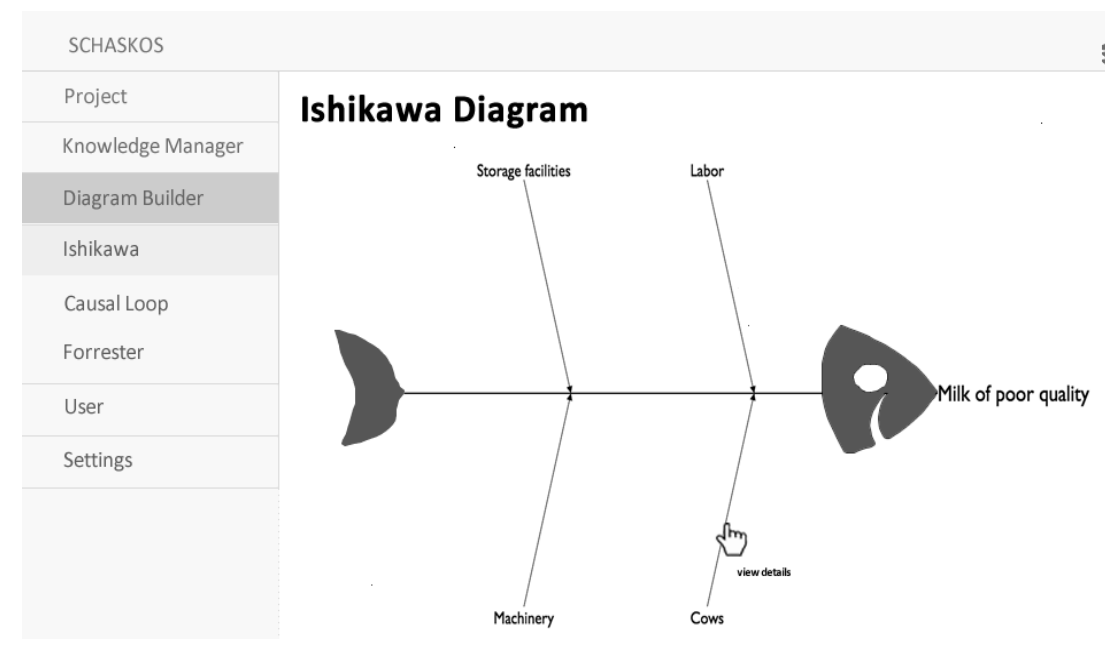

Fig. 2. Ishikawa Diagram generated for SCHASKOS.

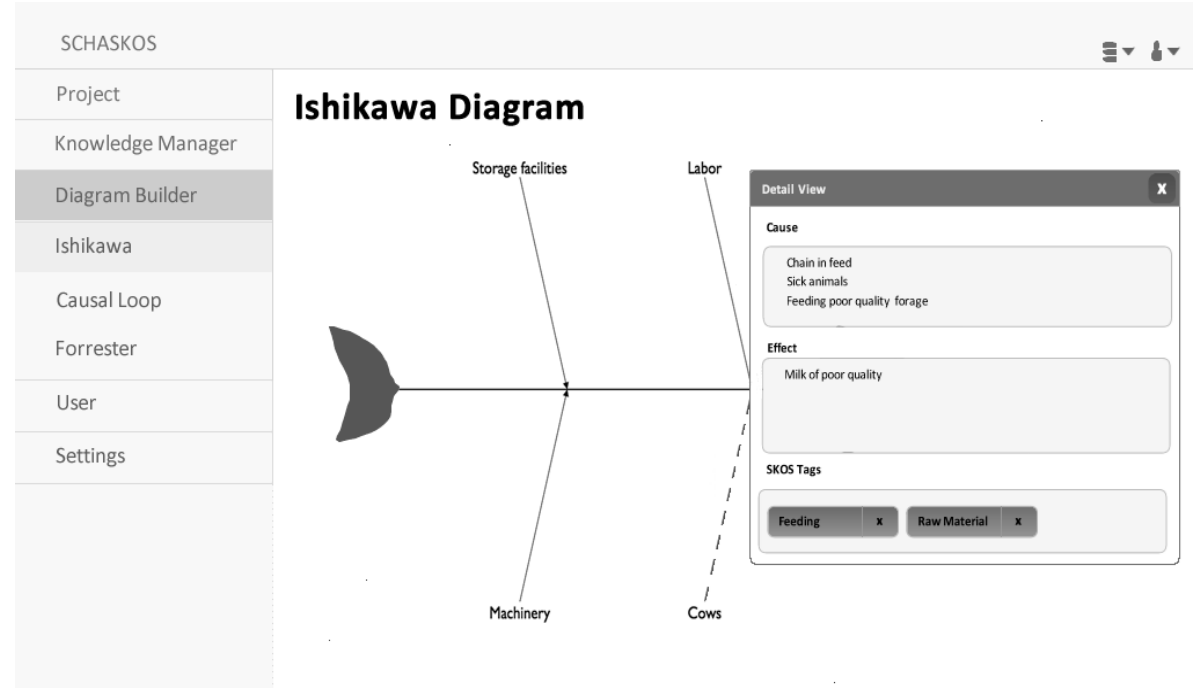

Fig. 3. Details of a cause in the Ishikawa diagram shown by SCHASKOS.

As shown in Figures 2 and 3, the Ishikawa diagram visualization tool provided by SCHASKOS presents a detail view modal window when clicking in a category of causes of the "Milk of poor quality" event. The Figure 3 shows in a dashed line, the selected category. In the modal window, different causes related with the "Cows" are shown. In this regard a side effect of feeding cows whit poor quality forage insides in Milk quality. 


\section{Conclusions and Future Work}

The knowledge has become a strategic asset for organizations, due to this; organizations must invest in developing the best strategy for applying the knowledge assets in their organizations. From this perspective, a knowledge organization system allows to organizations to manage data, and transform these data to business knowledge. In this context, SCHASKOS transform the information for the generation and development of Ishikawa, Causal or Forrester diagram to end-users, in order to provide a graphic tool for helping on the decision-making process. Finally, with the use of SKOS, the decision-making process can be improved with knowledge sharing and management in supply chains. As future work, we have considered to analyze the backward effects of decision making across the supply chain. Additionally, we propose to include the ability for connecting SCHASKOS with external data sources to extract the information and build other kind of diagrams. At the same time, a supply chain ontology in the tool will be developed in order to infer new knowledge that can be useful on the decision-making process.

\section{References}

1. Hafeez, K., Rodiguez-Falcon, E., Abdelmeguid, H., Malak, N., Training, P.: Knowledge Management in Supply Chains. In: ICSTM. (2000)

2. https://climate.iarc.uaf.edu/geonetwork/docs/eng/users/admin/index.html.

3. Lacasta, J., Nogueras-Iso, J., López-Pellicer, F.J., Muro-Medrano, P.R., Zarazaga-Soria, F.J.: ThManager: An open source tool for creating and visualizing SKOS. Information Technology and Libraries, Vol. 26, pp. 39-51 (2013)

4. Sibille-de Grimoüard, C.: The Thesaurus for French Local Archives and the Semantic Web. Procedia-Social and Behavioral Sciences, 147, pp. 206-212 (2014)

5. http://www.zora.uzh.ch/98982/22/Developing\%20a\%20Web\%E2\%80\%90based\%20SKOS $\% 20$ Editor.pdf.

6. http://aims.fao.org/vest-registry/tools/vocbench-2.

7. Cox, S., Yu, J., Rankine, T.: SISSVoc: A Linked Data API for access to SKOS vocabularies. Semant, Web (2014)

8. http://labs.sparna.fr/skos-play/about.

9. Morshed, A., Dutta, R.: Machine Learning Based Vocabulary Management Tool Assessment for the Linked Open Data. International Journal of Computer Applications, Vol. 60, (2012)

10. Croom, S., Romano, P., Giannakis, M.: Supply chain management: an analytical framework for critical literature review. European journal of purchasing \& supply management, Vol. 6, pp. 67-83 (2000)

11. Samuel, K.E., Goury, M.-L., Gunasekaran, A., Spalanzani, A.: Knowledge management in supply chain: An empirical study from France. The Journal of Strategic Information Systems, Vol. 20, pp. 283-306 (2011)

12. Marra, M., Ho, W., Edwards, J.S.: Supply chain knowledge management: A literature review. Expert Systems with Applications, Vol. 39, pp. 6103-6110 (2012)

13. Patil, S.K., Kant, R.: A fuzzy AHP-TOPSIS framework for ranking the solutions of Knowledge Management adoption in Supply Chain to overcome its barriers. Expert Systems with Applications, Vol. 41, pp. 679-693 (2014)

14. Huang, C.-C., Lin, S.-H.: Sharing knowledge in a supply chain using the semantic web. Expert Systems with Applications, Vol. 37, pp. 3145-3161 (2010)

15. Almuiet, M.Z., Salim, J.: Knowledge flow in supply chain manufacturing: Case study in food manufacturing firm. Procedia Technology, Vol. 11, pp. 463-470 (2013) 